\title{
Time Filtering Techniques for Echo Reduction in Antenna Measurements
}

\author{
Pilar González-Blanco . Manuel Sierra-Castañer
}

\begin{abstract}
This paper presents a review of the time filtering methods to eliminate echo in antenna measurements. Two different methods, FFT and Matrix Pencil, are compared and simulated in a Planar Near Field (PNF) where other effects, such as aliasing, can happen if the simulation is not carefully carried out. Finally both time gating methods are applied to different real measurement of several antennas in different antenna measurement systems.
\end{abstract}

Index Terms-antennas, measurement, time gating, matrix pencil, Fourier transform.

\section{INTRODUCTION}

Antenna pattern measurements are usually carried out to check that the antennas are actually working the way they were designed to. To perform the measurements, anechoic chambers are often used in order to avoid many undesired contributions, such as the diffractions or reflections that are present in open environments.

When fully anechoic conditions are not satisfied, wellestablished procedures for echo reduction have been developed. In this paper, we will focus mainly in time gating techniques, where the main idea is to separate the direct signal from the reflections using a time-domain representation. This information is used to gate the direct contribution and cancel the echo effects. There are different methods for performing this filtering, and, in this paper, the authors have used the wellknown Fourier Transform [1] and the Matrix Pencil methods [2-3] for the cancelation of the echoes in antenna tests. FFT method has been used in [4] with other modal filtering methods to improve the results of antenna measurements. However, the objective of the paper is to compare both methods and to analyze the limitations and advantages of each technique for antenna measurements

The paper is organized as follows. In Section II we review the time filtering techniques mentioned above. Some simulations of both methods and measurements performed in a real anechoic chamber are described in section III. Finally, conclusions are presented in section IV.

The authors want to acknowledge the Madrid Region Government project for financing the project Space Debris Radar (S2013/ICE-3000 SPADERADAR-CM) and the Spanish Government, for the support of the project ENABLING5G "Enabling Innovative Radio Technologies for $5 \mathrm{G}$ networks" (TEC2014-55735-C3-1-R)

\section{TIME FILTERING}

Historically, time filtering was the first method used for echo reduction. In this paper the authors will study two different methods for time filtering, the FFT method and the Matrix Pencil.

\section{A. $F F T$}

The first approach is the use of a FFT to pass from the frequency domain to the time domain. Firstly the samples measured at different frequencies are transformed to the time domain (via inverse FFT), then the echoes are filtered out in this domain, and finally a new FFT is applied to reconstruct the field. This technique is applied to near field antenna measurement in [1]

The main drawback is that the fast algorithms used to compute the FT and IFT require a constant frequency step. Thus, when there are frequency regions where the field varies quickly, a small step has to be employed over the entire bandwidth to obtain a good characterization in those regions. Therefore, the measurement time can increase considerably.

\section{B. Matrix Pencil}

A second approach for time filtering is based on the Matrix Pencil algorithm. The Matrix Pencil is a linear method to approach the problem of finding the best estimates of a signal from the noise-contaminated data.

The signal is represented as a sum of exponentials multiplied by a residue. The Matrix Pencil gives the sharp values for the parameters of the problem: $M$ (the number of exponentials), $\mathrm{R}_{\mathrm{i}}$ (Residues) and $\mathrm{z}_{\mathrm{i}}$ (exponential factors). This method works quite well both with uniform and non-uniform frequency samples. Similar to the application of the Matrix Pencil Method is the application of a non-uniform DFT in the solution of the frequency to time-domain transformation.

In both cases, the main disadvantage is that the acquisition has to be multifrequency to be able to apply these algorithms.

\section{SOME EFFEcts to Be CONSIDERED IN THE GATING}

In this paper, the echo will be modeled as the reflection at the measurement points located in front of the antenna, simulating the case of a planar antenna with large scattering situated in front of the antenna probe placed on a metallic plate. This section studies firstly the effect of the aliasing, 
secondly the effect of the filtering and lastly the possibility of performing the filtering in near field data or in far field data.

\section{A. Effect of Bandwith and Step}

Depending on the total bandwidth and the number of samples, there will be a maximum distance where the peaks coming from direct and reflected ray can be detected. This distance should be at most $\mathrm{c} * \mathrm{dt} * \mathrm{~N}$, being $\mathrm{dt}$ the inverse of the bandwidth and $\mathrm{N}$ the number of samples. If the signal or the echoes are present at a greater distance, they will be received in the next sweep, giving rise to an aliasing effect. So, for the simulation, the parameters (number of samples) should be taken appropriately in order to avoid the aliasing effect.

Next, a simulation is presented with range from 0.8 to 6 $\mathrm{GHz}$, with the same parameters for setup taken except for the distance between the AUT and the probe which has been set so that the signal appears at $3.6 \mathrm{~m}$ and the echo at $11 \mathrm{~m}$. As the maximum distance that one needs to consider is $11 \mathrm{~m}$, the minimum step should be the velocity of the light divided by maximum distance, which is in this case $27.27 \mathrm{MHz}$.

Fig. 1 shows some examples with different measurement bandwidth and step. As it is well known, the bandwidth is related to the resolution in time domain. Obviously, the resolution has to be good enough to separate the direct ray and the reflected ray. The second parameter is the frequency step. As discussed above, a minimum step of $27.27 \mathrm{MHz}$ is needed for avoiding the aliasing. This can be seen in the first row. However, in the other two cases, the information is still there. In the second row (50 MHz step), there is aliasing for the reflected ray: this means that the reflected rays do not appear in the correct position. However, the reflected ray can still be filtered out. However, in the third case (100 MHz step), the aliasing appears for both the direct and reflected ray, so in this case it is difficult to identify the direct ray.

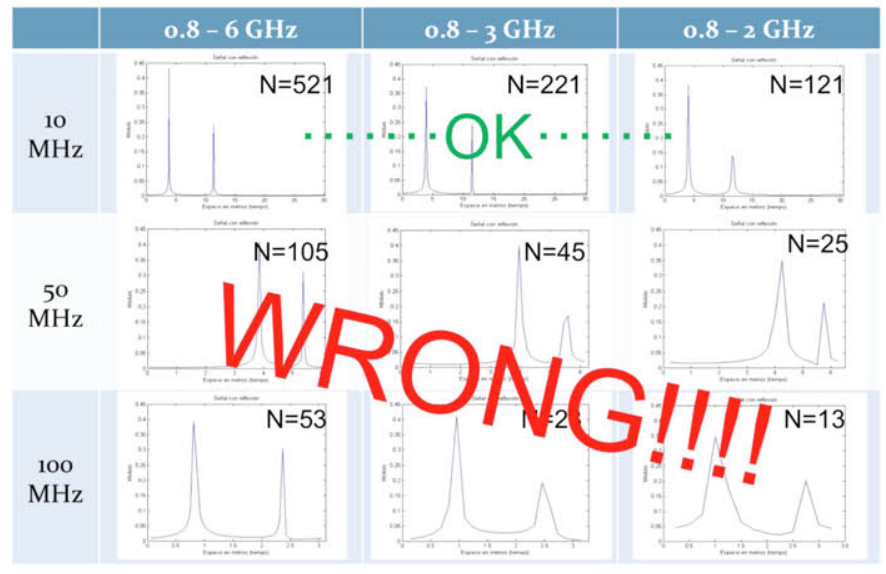

Fig. 1. Different combinations of bandwidth and frequency step

\section{B. Effect of different windows and techniques}

Two different techniques are used for the time gating: Fourier Transform and Matrix Pencil method. Fourier Transform requires a filtering in time domain, and several windows can be used in that filtering. In this paper, the rectangular and Hamming windows are analyzed, and the results are shown in Fig. 2. Matrix Pencil works in a different way, since the spurious rays are canceled after the detection of the respective exponentials. Fig. 3 shows the result of the application of Matrix Pencil to some of the cases shown in Fig. 1. It is observed that with a $10 \mathrm{MHz}$ step all the cases work perfectly.
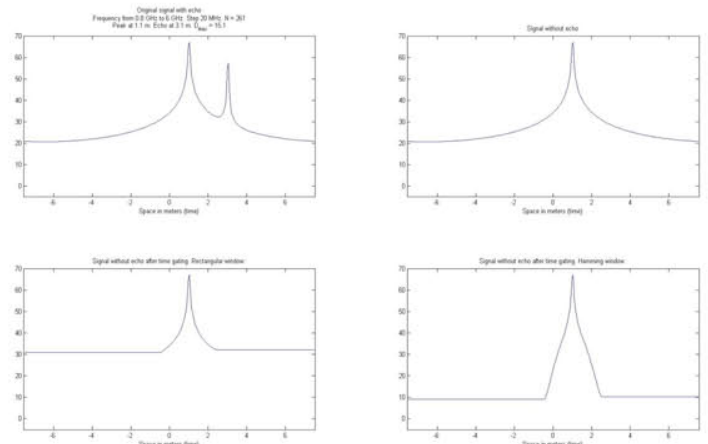

Fig. 2. Time gating based on FFT with rectangular and Hamming window

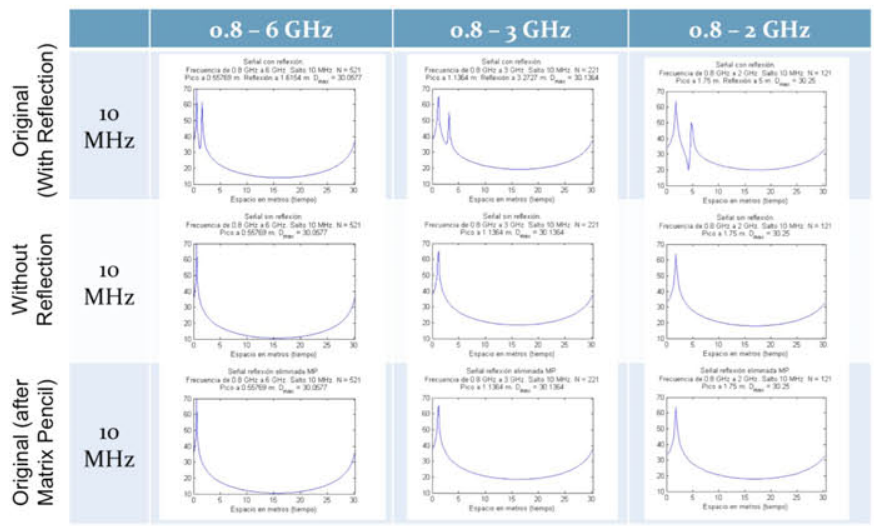

Fig. 3. Application of Matrix Pencil to the simulated reflection.

\section{Application to Antenna Measurements}

When these algorithms are applied to antenna measurements, some other difficulties appear. The main problem is the need for multifrequency measurements. This complicates the process for some cases as in the classical near field acquisition the measurement time can be very large. Of course, another aspect is the presence of noise. However, this is unusually very significant since the signal to noise ratio in the main lobes is large. In the case of planar or cylindrical near field systems another problem appears: the distance between AUT and probe is different for each measurement point. This implies that the time gating has to be adjusted for each measurement point. However, in the real situations the effect of the reflection is typically strongest when the probe is in front of the AUT (at least when the interactions between both antennas are dominant). In this case, the gating can be applied only to those points.

One advantage is that, since both the processes of near to far field transformation and time gating are linear, this time gating can be applied to the far fields. This simplifies the method and makes it independent of the antenna measurement 
system. These considerations will be discussed in detail in a future contribution

\section{MEASUREMENTS}

In order to validate the simulations, a slot antenna has been measured in the planar-range measurement system in the anechoic chamber at the Universidad Politécnica de Madrid (UPM). The antenna was designed and measured in X-band, from $7.7 \mathrm{GHz}$ to $8.6 \mathrm{GHz}$. The measured was taken at $25 \mathrm{~cm}$, acquiring 61 points in each axis, separated $2 \mathrm{~cm}$ each. The frequency step taken was $50 \mathrm{MHz}$. Also, a dipole antenna was measured in a StarLab system and a quadridge horn SH2000 in a single cut system in the facilities of Microwave Vision Italy (Fig. 4)

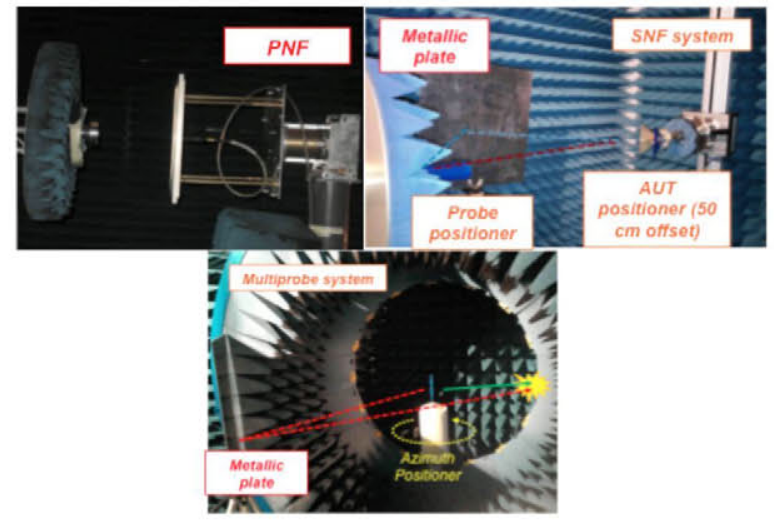

Fig. 4. Measurement Setups analyzed

Different plates have been employed in these setups. In the case of the PNF, a plate has been located behind the antenna probe. In the case of the single cut measurement the plate has been placed in the wall, and in the case of the multiprobe system in one side. Some of the results are displayed below. Fig. 5 shows the application of FFT and Matrix Pencil to a measurement in the planar near field system. It is observed that both algorithms can cancel the reflection.

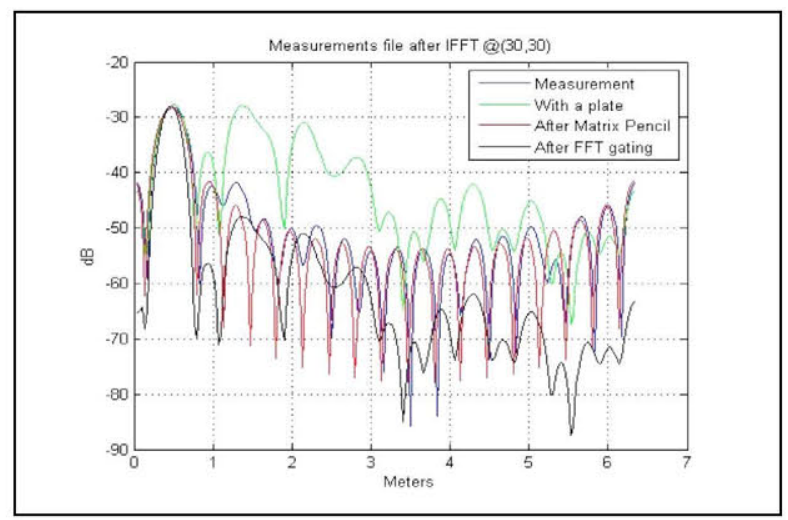

Fig. 5. Measurements after time gating with Matrix Pencil and FFT
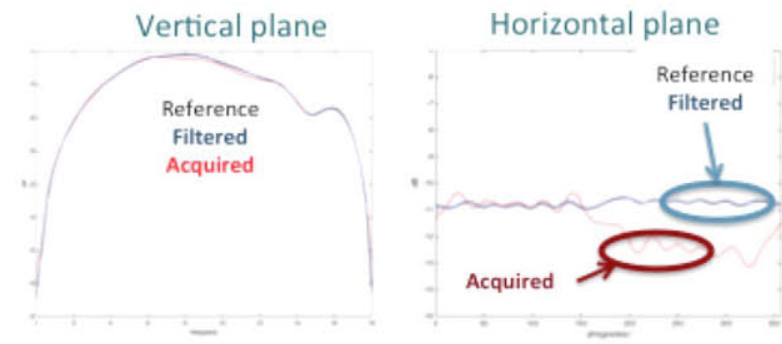

Fig. 6. Far Field patterns: reference, with reflection and after time gating (FFT) for the dipole antenna.

Fig. 6 shows the radiation patterns (horizontal and vertical) for one of the cases. In this case, the time gating has been applied on the far field transformed measurements, obtaining accurate results. There are still some oscillations because the effect of the positioner, which cannot be cancelled since is too close to the antenna dipole.

\section{CONCLUSIONS}

We have discussed the application of time gating through FFT and Matrix Pencil algorithms for the reduction of echoes in the measurement of antennas in different systems. The main drawback of the method is the requirement of multifrequency measurements. An analysis of the required number of samples has been performed. Also, the algorithms have been applied to different antenna measurement systems.

\section{ACKNOWLEDGMENT}

The authors would like to thank Microwave Vision Italy and LEHA-UPM antenna measurement laboratory for the antenna measurements that they have performed.

\section{REFERENCES}

[1] Aubin, J., Winebrand, M., Soerens, R., and Vinogradov, V., "Accurate near-field measurements using time-gating," Antenna Measurement Techniques Association Annual Symposium Proceedings, pp. 362-365, Nov. 2007.

[2] S. Loredo, M. R. Pino, F. Las-Heras, and T. K. Sarkar, "Echo identification and cancellation techniques for antenna measurement in non-anechoic test sites," IEEE Antennas Propagat. Mag., vol. 46, No. 1, pp. 100-107, Feb., 2004.

[3] T. K. Sarkar and O. Pereira, "Using the matrix pencil method to estimate the parameters of a sum of complex exponentials," IEEE Antennas Propagat. Mag., vol. 37, No. 1, pp. 48-55, Feb., 1995

[4] M. Sierra Castañer, P. González-Blanco García, M.J. López Morales, F. Saccardi, L. J. Foged. "Time and Spatial Filtering for Echo Reduction in Antenna Measurements". Proceedings of the AMTA Symposium 2015. Long Beach, CA, United States. October 2015. 